\title{
Electrochemical Determination of Catalpol in Rehmannia Glutinosa Based on Polyaniline-Graphene Modified Glassy Carbon Electrode
}

\author{
Xuemei Wang ${ }^{1,2 \#}$, Dike Zhou ${ }^{3 \#}$ and Yanchun Wang ${ }^{1,2 *}$ \\ ${ }^{1}$ People's Hospital of Zhengzhou University, Zhengzhou, 450003, Henan, P. R. China \\ ${ }^{2}$ Department of Traditional Chinese Medicine,Henan Provincial People's Hospital, Zhengzhou, \\ 450003, Henan, P. R. China \\ ${ }^{3}$ Department of Endocrinology, The First Affiliated Hospital of Zhengzhou University, Zhengzhou, \\ 450052, Henan, P. R. China \\ \#These authors contributed equally to this work \\ *E-mail: wangxuemei211@ sohu.com
}

doi: $10.20964 / 2017.06 .17$

Received: 19 September 2016 / Accepted: 28 March 2017 / Published: 12 May 2016

In this work, we prepared an electrochemical sensor to quantify the catalpol, where the glassy carbon electrode (GCE) was modified by the polyaniline graphene. Compared with the bare GCE, we found that the GCE modified with polyaniline graphene exhibited a well-defined oxidation of the catalpol. Moreover, a significant enhancement in the current response was observed during the process. Especially, the current response of the oxidation peak of the catalpol exhibited a linear relationship with the concentration of the catalpol in the range of 0.005 to $50 \mu \mathrm{M}$, where the limit of the detection was $0.002 \mu \mathrm{M}$. The electrochemical sensor was succeeded to be applied in the determination of the catalpol in the root of Rehmannia glutinosa the due to the improved voltametric performance.

Keywords: Rehmannia glutinosa; Catalpol; Polyaniline; Sensing; Electrode

\section{FULL TEXT}

(C) 2017 The Authors. Published by ESG (www.electrochemsci.org). This article is an open access article distributed under the terms and conditions of the Creative Commons Attribution license (http://creativecommons.org/licenses/by/4.0/). 